\title{
Three-Dimensional Imaging of Cerebellar Mossy Fiber Rosettes by Ion-Abrasion Scanning Electron Microscopy
}

\author{
Hyun-Wook Kim, ${ }^{1}$ Namkug Kim, ${ }^{2}$ Ki Woo Kim, ${ }^{3, *}$ and Im Joo Rhyu ${ }^{1, \star}$ \\ ${ }^{1}$ Department of Anatomy, College of Medicine, Korea University, 126-1 Anam dong 5 ka SungBuk Ku, Seoul 136-705, Korea \\ ${ }^{2}$ Department of Radiology, Research Institute of Radiology, Asan Medical Center, University of Ulsan College of Medicine, \\ 388-1 Pungnap-dong, Songpa-gu, Seoul 138-736, Korea \\ ${ }^{3}$ School of Ecology and Environmental System, Kyungpook National University, Sangju 742-711, Korea
}

\begin{abstract}
The detailed knowledge of the three-dimensional (3D) organization of the nervous tissue provides essential information on its functional elucidation. We used serial block-face scanning electron microscopy with focused ion beam (FIB) milling to reveal 3D morphologies of the mossy fiber rosettes in the mice cerebellum. Three-week-old C57 black mice were perfused with a fixative of $1 \%$ paraformaldehyde/1\% glutaraldehyde in phosphate buffer; the cerebellum was osmicated and embedded in the Araldite. The block containing granule cell layer was sliced with FIB and observed by field-emission scanning electron microscopy. The contrast of backscattered electron image of the block-face was similar to that of transmission electron microscopy and processed using 3D visualization software for further analysis. The mossy fiber rosettes on each image were segmented and rendered to visualize the 3D model. The complete 3D characters of the mossy fiber rosette could be browsed on the A-Works, in-house software, and some preliminary quantitative data on synapse of the rosette could be extracted from these models. Thanks to the development of two-beam imaging and optimized software, we could get 3D information on cerebellar mossy fiber rosettes with ease and speedily, which would be an additive choice to explore 3D structures of the nervous systems and their networks.
\end{abstract}

Key words: cerebellum, mossy fiber rosette, three dimension, ion-abrasion SEM

\section{INTRODUCTION}

The detailed knowledge of the three-dimensional (3D) organization of the biological tissue provides essential information on its functional elucidation, especially in the nervous system. Since the first report of transmission electron microscopy (TEM) of the cerebral cortex synapse (Gray, 1959), TEM has contributed to the accomplishment of neuroscience. 3D ultrastructural analyses on the small part of the mammalian brain and the complete nervous system on Caenorhabditis elegans (White et al., 1986) have shed significant light on 3D microscopic study. The redistribution of polyribosome on the dendritic shaft and their spines in long-term potentiation slice of the rat hippocampus with 3D TEM study revealed the importance of the local protein synthesis during learning and memory (Ostroff et al., 2002). In addition, a lot of effort is being made to perform largescale 3D analysis of neural connectivity with various modern microscopic technologies (Eisenstein, 2009).

Three methods are available for 3D ultrastructural analyses of the nervous system: (1) serial section transmission electron microscopy (SSTEM), (2) serial block-face scanning electron microscopy (SBFSEM), and (3) serial section electron tomography (SSET) (Arenkiel \& Ehlers, 2009). The SSTEM is a widespread tool used in many classical studies. There are many factors that can contribute to interrupted series or imperfect image stacks: section folds during the placement onto support films, electron beam damage that

() MICROSCOPY SOCIETY OF AMERICA 2013

${ }^{\star}$ Corresponding authors. E-mail: kiwoo@knu.ac.kr; irhyu@korea.ac.kr distorts the sections, and stain contamination, which is the reason for the few reports on 3D microscopic studies in contrast to the long history of TEM. The SSET provides higher $z$-axis resolution than SSTEM and SBFSEM (Noske et al., 2008), but requires high-end manipulating skills of imaging process. SBFSEM has been introduced recently thanks to two combining technologies: (1) serial block milling methods by diamond knife or ion beam and (2) detection system of backscattered electron signals (Denk \& Horstmann, 2004; Knott et al., 2008). SBFSEM is operated unattended, alleviating many problems associated with SSTEM, automatically generating aligned serial images (Knott et al., 2008; Kim et al., 2010). Focused ion beam (FIB) systems have emerged as powerful tools for biological materials, as well as metals and semiconductors (Hou \& Yao, 2007). A modern modality of FIB having a gallium ion $\left(\mathrm{Ga}^{+}\right)$source is commonly equipped with a field-emission scanning electron microscope (FESEM), ensuring a twobeam system (FIB-FESEM). With its in situ machining and high-resolution imaging abilities, FIB-FESEM can provide novel applications that were not available through conventional microscopy. There is growing evidence that $3 \mathrm{D}$ reconstructions of complex biological structures can be implemented using FIB-FESEM (Bushby et al., 2011).

The mossy fiber rosettes are a core structure forming cerebellar glomeruli, major excitatory synapse to cerebellar granule cells, and Golgi cells (Palay \& Chan-Palay, 1974). Mossy fiber rosettes are a large synapse complex of several micrometers in diameter contacting as many as $\mathbf{5 0}$ granule cells (Jakab \& Hamori, 1988; Jakab, 1989). The construction 
of the 3D models of the mossy fiber rosettes required a number of serial TEM section images from 97 to 177 (Xu-Friedman \& Regehr, 2003).

Here, we applied FIB-FESEM to model 3D morphology of the cerebellar mossy fiber rosettes and extracted some morphological characters of the mossy fiber rosettes, which can be compared with those under various physiological and pathological conditions in specific cases.

\section{Materials and Methods}

\section{Sample Preparation}

Three-week-old C57BL mice were anesthetized with ketamine/xylazine (ip) and perfused intracardially with $250 \mathrm{ml}$ of $1 \%$ paraformaldehyde/1\% glutaraldehyde in $0.12 \mathrm{M}$ phosphate buffer $(\mathrm{pH}$ 7.4). The cerebella were dissected and 0.5-1-mm sagittal sections of each cerebellum were osmicated ( $2 \%$ osmium tetroxide) for $3-4 \mathrm{~h}$, washed in maleic acid buffer, placed in a uranyl acetate coloration solution $\left(4^{\circ} \mathrm{C}\right)$ for $90 \mathrm{~min}$, washed again in maleic acid buffer, dehydrated through an ascending series of ethanol, and embedded in Araldite. The granule cell layer was identified on the semithin section and trimmed for further observation.

\section{FIB-FESEM}

The selected blocks were mounted on an aluminum stub and sputter-coated (ca. $10 \mathrm{~nm}$ thick) with platinum $(\mathrm{Pt})$ using a coater (SCD 005; BAL-TEC, Balzers, Liechtenstein). They were mounted on a stage of an FIB-FESEM (Auriga; Carl Zeiss, Oberkochen, Germany) and tilted to an angle of $54^{\circ}$. After Pt deposition for protection layer, the blocks were treated with a 4-nA (coarse milling) $\mathrm{Ga}^{+}$beam and a 240-pA (fine milling) $\mathrm{Ga}^{+}$beam operated at an acceleration voltage of $30 \mathrm{kV}$. The blocks were continuously sliced by $80 \mathrm{~nm}$ with $\mathrm{Ga}^{+}$beam, leading to a total of 283 slices (ca. $19,810 \mathrm{~nm}$ thick). The newly exposed surface was observed using an energy-selective backscattered electron detector at an acceleration voltage of $1.5 \mathrm{kV}$. It took ca. 3 min per slice-and-image step, a total of $849 \mathrm{~min}(14.15 \mathrm{~h})$. Acquired images were contrast-inverted using a built-in software package (SmartSEM version 5.3.5; Carl Zeiss, Oberkochen, Germany).

\section{D Image Processing}

Alignment of the contrast-inverted images was performed with Adobe Photoshop (Adobe Systems, San Jose, CA). Two adjacent images were overlaid in layers by comparing and aligning the bottom layer with the top layer (reference image) to make the reference image partially transparent (Knott et al., 2008). After alignment, the serial images were imported using in-house software, A-View software (developed at radiology department in Asan Medical Center), which was originally developed for 3D image visualization of CT and MRI images (Park et al., 2009). The original program was patched to import serial tiff format images produced by FIB-FESEM. The mossy fiber rosettes and their

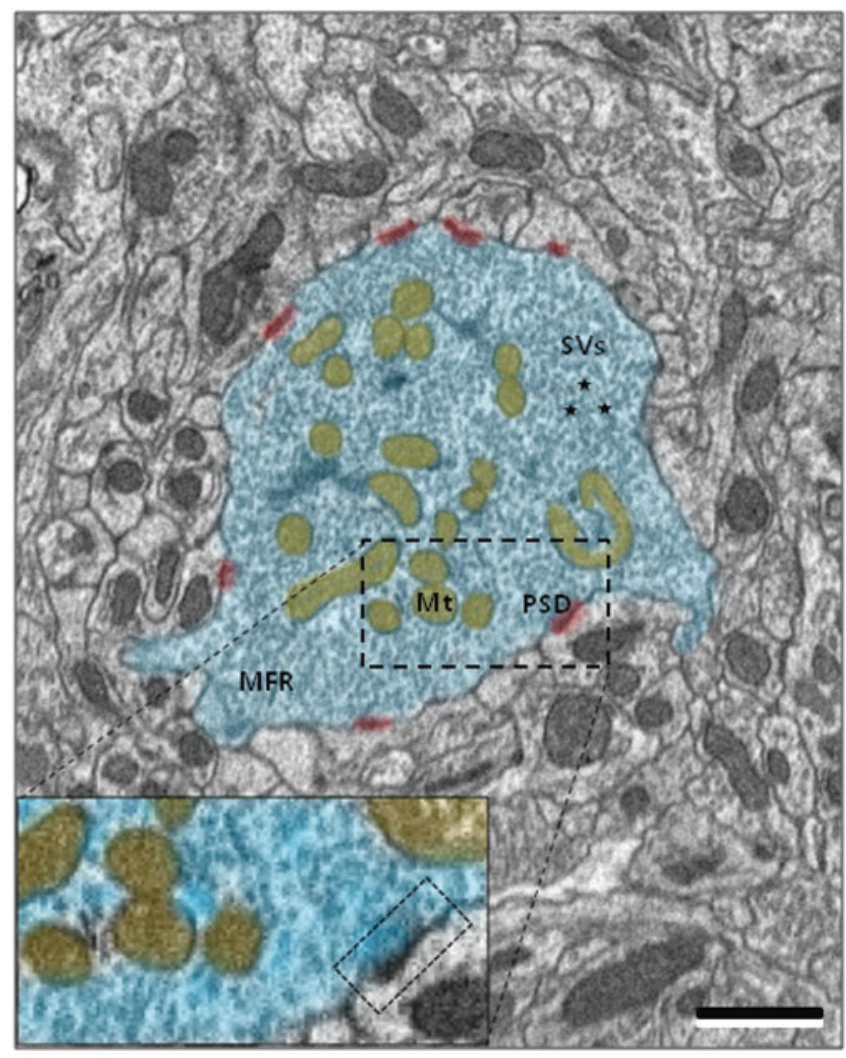

Figure 1. Ultrastructure of the mossy fiber rosette (MFR) on the milled surface of the block (MFR; sky blue). The MFR includes many synaptic vesicles (SVs, $\star$ ) and mitochondria (Mt; yellow). The electron-dense areas are synaptic contacts with postsynaptic components, which are marked as a red-colored area (PSD; red). Scale bar is $1 \mu \mathrm{m}$.

subcomponents were identified, segmented, and rendered to produce 3D models of the mossy fibber rosette. In addition, we measured some preliminary parameters such as volume of mossy fiber rosettes and their synaptic contact number.

\section{RESULT}

The block-face of the epoxy blocks was sliced with $\mathrm{Ga}^{+}$ beam by FIB-FESEM without discernible beam damage. Cellular membrane systems were considered preserved on the exposed block-face. In particular, it was feasible to resolve osmicated and pre-stained membranes by the compositional contrast of backscattered electron images (Kim \& Jaksch, 2009).

In this study, we milled the block by $80 \mathrm{~nm}$ steps to save milling time. It took $14 \mathrm{~h}$ to mill and image 283 times. The 283 serial images showed continuous change of the neuronal tissue profiles across the granule cell layer. When we aligned two images with Adobe Photoshop, there was a little shift of image in the $x-y$ axes. The contrast-inverted backscattered electron images of the block-face were very similar to conventional TEM images. We could identify all neuronal profiles including cell bodies, axon, dendrites, detailed organelles, and synapses (Fig. 1). The mossy fiber rosette 

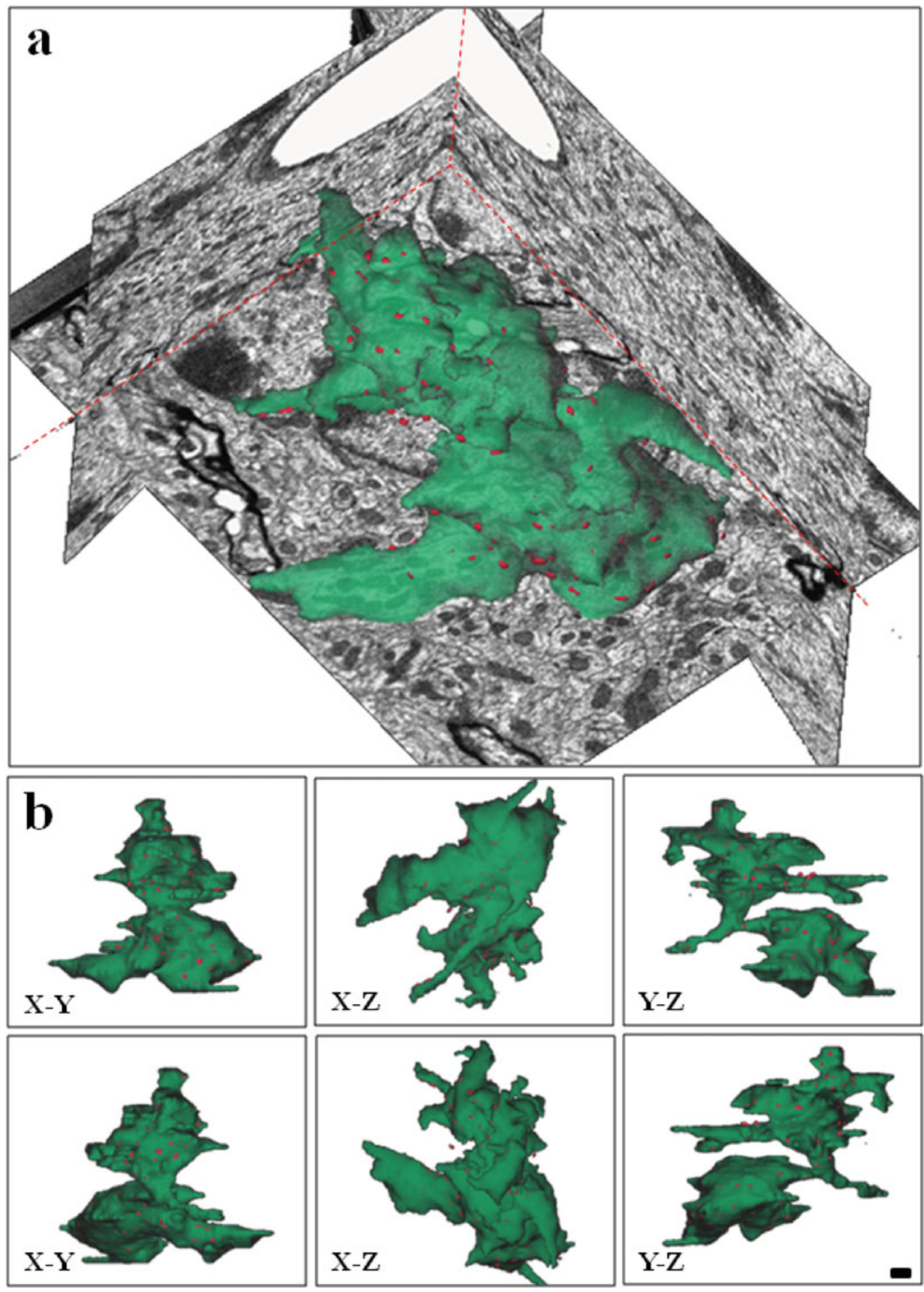

Figure 2. Screen capture images of a 3D model of mossy fiber rosette on the V-View program. The mossy fiber rosette can be observed on the 3D coordinate planes (a). The 3D model shows different projection images according to the six-window directions of the mossy fiber in the cube (b), which suggests the importance of $3 \mathrm{D}$ observations to understand real configuration of the biological structures. Surface of mossy fiber rosette, green; synaptic contact, red. Scale bar is $1 \mu \mathrm{m}$.

contains numerous synaptic vesicles, mitochondria, neurofilaments, and synapses contacting dendritic claws of granule cells and dendrites of Golgi cells (Fig. 1).

We constructed 3D models of mossy rosettes, which could be navigated in A-Works software (Fig. 2). A mossy fiber rosette was embedded in the virtual neuronal block correlated with its $x-y-z$ plane images (Fig. 2a). It had two lobules with isthmus and each lobule had irregular branched elongations (Figs. 2a, 2b). The morphology of this mossy fiber rosette is shown with different projections according 


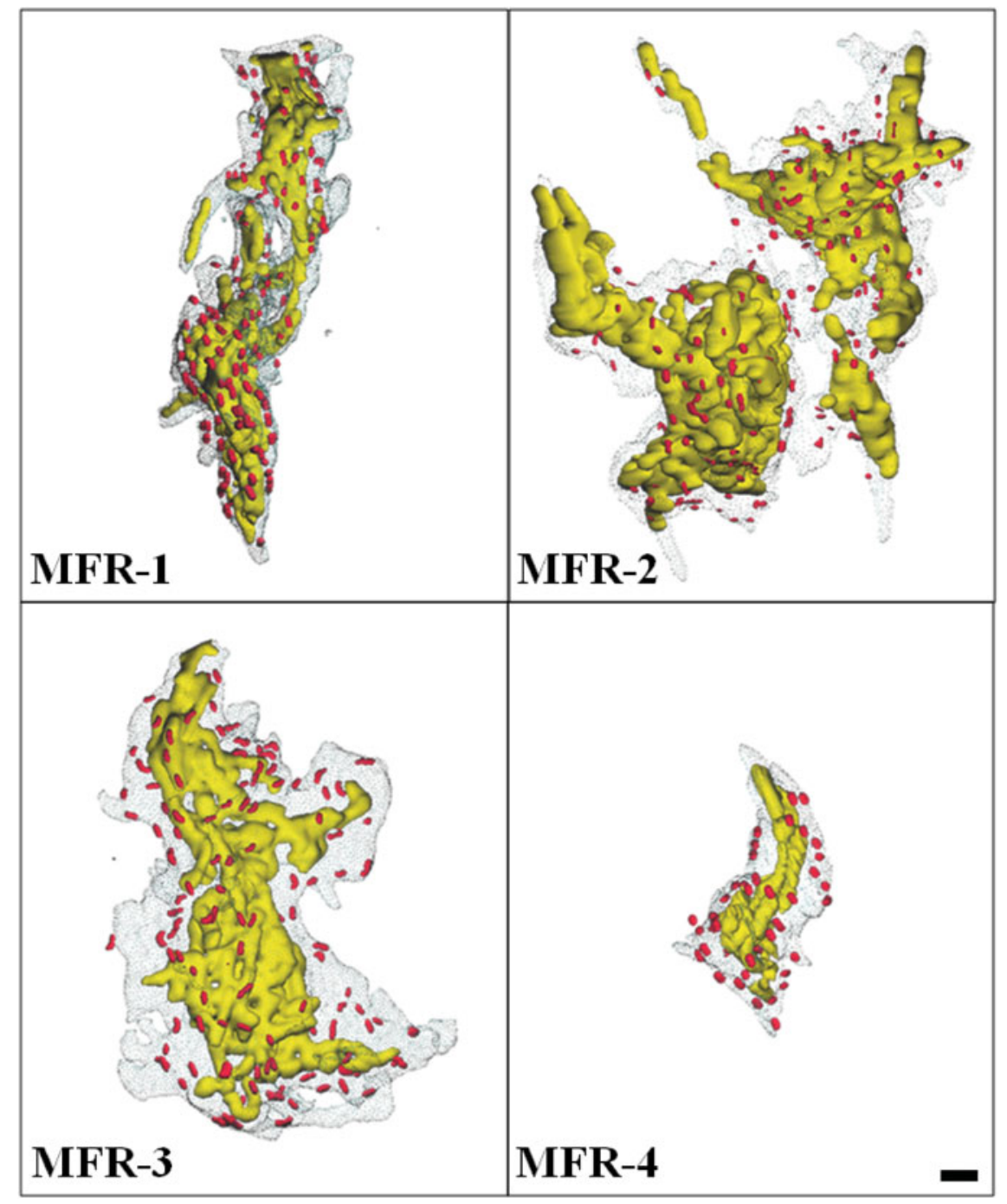

Figure 3. Three-dimensional models of mossy fiber rosettes (MFRs). The MFRs have diverse morphologies from simple fusiform (MFR-4) to complex lobulation with tubular processes (MFR-2). There are numerous mitochondria (yellow) inside of the rosettes and many synaptic contacts (red) observed on the surface of the MFRs. Scale bar is $1 \mu \mathrm{m}$. to its six observation windows (Fig. 2b). We constructed three and one incomplete model of mossy fiber rosette (Fig. 3). We mapped the location of synapses and distribution of mitochondria in each mossy fiber model. The models showed that there were large volume fractions of the mitochondria and synaptic contacts on the mossy fiber rosettes (Fig. 3).

The preliminary measurement of these mossy fiber rosettes model showed that the volume of the completely modeled mossy fiber rosettes was $31.2,36.7$, and $60.3 \mu \mathrm{m}^{3}$ (Table 1). The number of synaptic contact in each mossy fiber rosette was 140, 160, and 243 in case of complete sets. The synaptic contact number based on each volume was $4.03,4.36$, and 4.48 for all models reconstructed. The volume of the mitochondrial volume fraction ranged from 13.9 to $17.8 \%$.

\section{Discussion}

In this study, we efficiently reconstructed mossy fiber rosettes three dimensionally using SBFSEM images, which facilitated the alignment compared to SSTEM or SSET by

Table 1. Some Preliminary Measurement of Reconstructed 3D Mossy Fiber Rosette (MFR).

\begin{tabular}{lcccc}
\hline & \multicolumn{3}{c}{ MFR } \\
\cline { 2 - 5 } Contents & No. 1 & No. 2 & No. 3 & No. 4 \\
\hline Volume of MFR $\left(\mu \mathrm{m}^{3}\right)$ & 36.6739 & 60.2304 & 31.2503 & 9.37 \\
Number of PSD & 160 & 243 & 140 & 42 \\
Number of PSD/unit volume $\left(\mu \mathrm{m}^{3}\right)$ & 4.3628 & 4.0345 & 4.48 & 4.4824 \\
Volume of mitochondria $\left(\mu \mathrm{m}^{3}\right)$ & 5.5676 & 10.7329 & 4.1852 & 1.3802 \\
Percentage of mitochondria/MFR & 15.1815 & 17.8198 & 13.3925 & 14.7303 \\
\hline
\end{tabular}


having a fixed block surface. The quality of serial block-face image based on backscattered electron imaging was comparable to that of TEM (Fig. 1), which corresponded to the previous studies on nervous system (Knott et al., 2008; Merchan-Perez et al., 2009).

Two types of SBFSEM are being used for 3D microscopy. One is the two-beam system using $\mathrm{Ga}^{+}$ion abrasion as in this study (Merchan-Perez et al., 2009). The other is the FESEM equipped with an ultramicrotome inside of the SEM (Denk \& Horstmann, 2004), which is a commercially available model called 3View ${ }^{\circledast}$ (Gatan, CA, USA). The 3View ${ }^{\circledast}$ has some advantages compared with the ion-abrasion system: (1) wider milling area, (2) rapid milling speed, and (3) economic maintenance compared with $\mathrm{Ga}^{+}$system (Muller-Reichert et al., 2010). However, the ion-abrasion system (IA-SEM) has a higher $z$-axis resolution compared with the $3 \mathrm{View}^{\oplus}$ system. IA-SEM can mill some intractable specimens, which are not easy to cut with a diamond knife by routine preparations: bone/dental implant interfaces (Giannuzzi et al., 2007), mannitol particle in inhalation aerosol (Heng et al., 2007), and oyster shell (Kudo et al., 2010). Therefore, we can choose an appropriate system according to samples observed.

We used A-Works, which was originally developed for $3 \mathrm{D}$ visualization of medical images such as CT and MRI. We successfully imported serial images and segmented, edited, and modeled by convenient user-friendly tools embedded in the software. Thanks to the combination of decent serial imaging system and user-friendly software, we could model mossy fiber rosettes efficiently.

The mossy fibers are axon fibers originating from the spinal cord or pons, and they make a unique branching pattern like Spanish moss. The enlargements of the mossy fibers are called rosettes, the morphology of which could be categorized into three groups: (1) simple fusiform enlargement, (2) massive central expansion with several twisted and angular, tapering, or filiform appendage projections, and (3) flower-like figures by tubular processes (Palay \& ChanPalay, 1974). In this study, we observed diverse 3D morphology of the mossy fiber rosettes corresponding to the above description. We could present some morphometric value on mossy fiber rosettes with 3D analysis. The number of synaptic contact per cubic micrometer was $4.0-4.5 / \mu \mathrm{m}^{3}$ and the volume fraction of mitochondria was $13.4-17.8 \%$ of the rosette volume, which could be used as reference values for further analysis of the synaptic strength in various physiological and pathological models.

Morphological differentiation of mossy fiber synapse was analyzed by developmental quantitative studies on the numerous random thin sections of the developing rat cerebella (Hamori \& Somogyi, 1983). The number of dendritic profiles belonging to mossy fiber rosette increased with the maturation of the cerebellum up to postnatal 60 days. The area and perimeter of the mossy fiber rosette area also showed an abrupt increase at postnatal 15 days. A hypothesis on the regulation of excessive number of synapse during development needs to be evaluated by $3 \mathrm{D}$ studies, because mossy fiber rosettes are big structures composed of over a hundred thin sections (Hamori \& Somogyi, 1983). The 3D models of cerebellar mossy fiber rosettes in a postnatal day 18 rat constructed by serial TEM provided morphological evidence of glutamate spillover caused by the desensitization within the mossy fiber and granule cell synapse (Xu-Friedman \& Regehr, 2003).

In addition to the mossy fiber rosette study, 3D modeling of the synapse in the LTP model of hippocampus implied an important role of multiple spine synapses between a single axon terminal and a dendrite during learning processes (Toni et al., 1999). The hypothesis of multiple spine formation in the LTP model was evaluated by SSTEM 3D analysis as well (Fiala et al., 2002). Recently, high throughput analyses of the neural network became an important platform for neuroscience, which is called "connectomics."

This study presented the potential of the two-beam applications for the reconstruction of the nervous system, which would be used for the analyses of normal neuronal structures and plasticity related to various neurological conditions.

\section{ACKNOWLEDGMENTS}

This work was supported by Basic Science Research Program through the National Research Foundation (NRF) funded by the Ministry of Education, Science and Technology (2010-0012753), and The Support Program for the Advancement of National Research Facilities and Equipment.

\section{REFERENCES}

Arenkiel, B.R. \& Ehlers, M.D. (2009). Molecular genetics and imaging technologies for circuit-based neuroanatomy. Nature 461(7266), 900-907.

Bushby, A.J., P’Ng, K.M., Young, R.D., Pinali, C., Knupp, C. \& QuANTOCK, A.J. (2011). Imaging three-dimensional tissue architectures by focused ion beam scanning electron microscopy. Nat Protoc 6 (6), 845-858.

Denk, W. \& Horstmann, H. (2004). Serial block-face scanning electron microscopy to reconstruct three-dimensional tissue nanostructure. PLoS Biol 2(11), e329.

Eisenstein, M. (2009). Neural circuits: Putting neurons on the map. Nature 461(7267), 1149-1152.

Fiala, J.C., Allwardt, B. \& Harris, K.M. (2002). Dendritic spines do not split during hippocampal LTP or maturation. Nat Neurosci 5(4), 297-298.

Giannuzzi, L.A., Phifer, D., Giannuzzi, N.J. \& Capuano, M.J. (2007). Two-dimensional and 3-dimensional analysis of bone/ dental implant interfaces with the use of focused ion beam and electron microscopy. J Oral Maxillofac Surg 65(4), 737-747.

Gray, E.G. (1959). Electron microscopy of synaptic contacts on dendrite spines of the cerebral cortex. Nature 183(4675), 1592-1593.

Hamori, J. \& Somogyi, J. (1983). Differentiation of cerebellar mossy fiber synapses in the rat: A quantitative electron microscope study. J Comp Neurol 220(4), 365-377.

Heng, D., Tang, P., Cairney, J.M., Chan, H.K., Cutler, D.J., Salama, R. \& Yun, J. (2007). Focused-ion-beam milling: A 
novel approach to probing the interior of particles used for inhalation aerosols. Pharm Res 24(9), 1608-1617.

Hou, K. \& YAO, N. (2007). Applications for Biological Materials. In Focused Ion Beam Systems. Cambridge, UK: Cambridge University Press.

JAKAB, R.L. (1989). Three-dimensional reconstruction and synaptic architecture of cerebellar glomeruli in the rat. Acta Morphol Hung 37(1-2), 11-20.

JAKAB, R.L. \& HAMORI, J. (1988). Quantitative morphology and synaptology of cerebellar glomeruli in the rat. Anat Embryol (Berl) 179(1), 81-88.

Kim, K., Baek, S., Park, B., Kim, H. \& Rhyu, I. (2010). Applications of focused ion beam for biomedical resarch. Kor J Microsc 40(4), 177-183.

KIM, K.W. \& JAKsCH, H. (2009). Compositional contrast of uncoated fungal spores and stained section-face by low-loss backscattered electron imaging. Micron 40(7), 724-729.

Knott, G., Marchman, H., Wall, D. \& Lich, B. (2008). Serial section scanning electron microscopy of adult brain tissue using focused ion beam milling. J Neurosci 28(12), 2959-2964.

Kudo, M., Kameda, J., Saruwatari, K., Ozaki, N., Okano, K., Nagasawa, H. \& Kogure, T. (2010). Microtexture of larval shell of oyster, Crassostrea nippona: A FIB-TEM study. J Struct Biol 169(1), 1-5.

Merchan-Perez, A., Rodriguez, J.R., Alonso-Nanclares, L., Schertel, A. \& Defelipe, J. (2009). Counting synapses using FIB/SEM microscopy: A true revolution for ultrastructural volume reconstruction. Front Neuroanat 3, 18.
Muller-Reichert, T., Mancuso, J., Lich, B. \& McDonald, K. (2010). Three-dimensional reconstruction methods for Caenorhabditis elegans ultrastructure. Methods Cell Biol 96, 331-361.

Noske, A.B., Costin, A.J., Morgan, G.P. \& Marsh, B.J. (2008). Expedited approaches to whole cell electron tomography and organelle mark-up in situ in high-pressure frozen pancreatic islets. J Struct Biol 161(3), 298-313.

Ostroff, L.E., Fiala, J.C., Allwardt, B. \& Harris, K.M. (2002). Polyribosomes redistribute from dendritic shafts into spines with enlarged synapses during LTP in developing rat hippocampal slices. Neuron 35(3), 535-545.

Palay, S. \& Chan-Palay, V. (1974). Cerebellar Cortex: Cytology and Organization. New York: Springer.

Park, I.S., Lee, K.J., Han, J.W., Lee, N.J., Lee, W.T., Park, K.A. \& RHyu, I.J. (2009). Experience-dependent plasticity of cerebellar vermis in basketball players. Cerebellum 8(3), 334-339.

Toni, N., Buchs, P.A., Nikonenko, I., Bron, C.R. \& Muller, D. (1999). LTP promotes formation of multiple spine synapses between a single axon terminal and a dendrite. Nature 402 (6760), 421-425.

White, J.G., Southgate, E., Thomson, J.N. \& Brenner, S. (1986). The structure of the nervous system of the nematode Caenorhabditis elegans. Phil Trans R Soc Lond B 314(1165), 1-340.

Xu-Friedman, M.A. \& Regehr, W.G. (2003). Ultrastructural contributions to desensitization at cerebellar mossy fiber to granule cell synapses. J Neurosci 23(6), 2182-2192. 